Original Article

\title{
Invasiveness and Malignant Potential of Pulmonary Lesions Presenting as Pure Ground-Glass Opacities
}

\author{
Junji Ichinose, MD, ${ }^{1}$ Tadasu Kohno, MD, ${ }^{1}$ Sakashi Fujimori, MD, ${ }^{1}$ \\ Takashi Harano, MD, ${ }^{1}$ Souichiro Suzuki, MD, ${ }^{1}$ and Takeshi Fujii, MD ${ }^{2}$
}

\begin{abstract}
Purpose: We retrospectively investigated the pathological diagnoses of pulmonary lesions presenting as pure ground-glass opacities (GGOs) to evaluate the risk of invasive malignancy. Methods: We examined 191 GGO lesions, including 114 pure GGO and 77 mixed lesions, in 160 patients who underwent resection between January 2008 and December 2010.

Results: Of the 114 pure GGO lesions, 14 (12\%) were diagnosed as invasive lung cancer and $16(14 \%)$ as minimally invasive adenocarcinoma. Twenty-one lesions exhibited pleural indentation on high-resolution computed tomography (HRCT), and 5 of these were diagnosed as invasive cancer, indicating an invasive tendency of pure GGO lesions with pleural indentation (odds ratio, 2.64). Of 14 pure GGO lesions positive on positron emission tomography (PET), 8 were diagnosed as invasive lung cancer, indicating an invasive tendency of pure GGO lesions with PET positivity (odds ratio, $16.0 ; p<0.001$; sensitivity, $67 \%$; specificity, $89 \%$ ).

Conclusion: Invasive lung cancer accounted for $12 \%$ of the pure GGO lesions. Pure GGO lesions should be carefully monitored by periodic chest computed tomography, and surgical resection is recommended when they exhibit pleural indentation on HRCT or positivity on PET.
\end{abstract}

Keywords: ground-glass opacity, adenocarcinoma in situ, minimally invasive adenocarcinoma, high-resolution computed tomography, positron emission tomography

\section{Introduction}

According to recent evaluations of sublobar resection and selective lymph node dissection, imaging technologies such as high-resolution computed tomography (HRCT), magnetic resonance imaging (MRI), and positron

${ }^{1}$ Department of Thoracic Surgery, Toranomon Hospital, Minato-ku, Tokyo, Japan

${ }^{2}$ Department of Pathology, Toranomon Hospital, Minato-ku, Tokyo, Japan

Received: February 17, 2013; Accepted: May 28, 2013

Corresponding author: Junji Ichinose, MD. 3-32-15 Horinouchi, Suginami-ku, Tokyo 166-0013, Japan

Email: i_junji@ba2.so-net.ne.jp

(C)2014 The Editorial Committee of Annals of Thoracic and Cardiovascular Surgery. All rights reserved. emission tomography (PET) play an important role in the selection of surgical treatment of lung cancer. Multiple studies ${ }^{1-7)}$ have shown a $100 \%$ 5-year disease-free survival rate, if complete resection is performed for patients with solitary lung adenocarcinoma exhibiting pure lepidic growth $(<2-3 \mathrm{~cm})$. Corresponding to adenocarcinoma in situ (AIS) in the International Association for the Study of Lung Cancer/American Thoracic Society/ European Respiratory Society international multidisciplinary classification of lung adenocarcinoma, ${ }^{8)}$ these tumors are a good indication for sublobar resection. Minimally invasive adenocarcinoma (MIA) is defined as a small, solitary adenocarcinoma exhibiting a predominantly lepidic growth pattern and $\leq 5 \mathrm{~mm}$ invasion in the greatest dimension of any one plane. Several reports ${ }^{9-13)}$ have described patients with similarly defined lung MIA 
and a disease-specific survival rate of nearly $100 \%$. Furthermore, some reports ${ }^{14,15)}$ have demonstrated that tumor malignancy depends on the maximum diameter or area of its solid component, excluding the ground-glass opacity (GGO) component. Correlations between tumor malignancy and MRI and PET findings have also been reported. ${ }^{16-18)}$ Consequently, the validity of sublobar resection is now under investigation in clinical trials.

In this study, we retrospectively investigated the pathological diagnoses of pulmonary lesions presenting as pure GGOs on HRCT to evaluate the potential risk of invasive malignancy. If tumor malignancy is determined by the size of its solid component, the question of whether pure GGO lesions can be treated as benign tumors arises. Because HRCT detects numerous tiny GGO lesions, a majority of which exhibit no changes over many years, pure GGO lesions are minimized. We also attempted to identify risk factors for invasive lung cancer from HRCT and PET findings.

\section{Materials and Methods}

Between January 2008 and December 2010, 1065 patients underwent pulmonary resection at Toranomon Hospital, Tokyo, Japan. From these, we retrospectively reviewed the medical records of 160 patients who underwent resection for GGO lesions $\leq 20 \mathrm{~mm}$ in diameter. We also investigated the presence of pleural indentation on HRCT and the maximum standardized uptake value (SUVmax) on PET.

GGO was defined as a hazy increase in lung attenuation without obscuring underlying vascular markings in the lung window on HRCT. Tumor disappearance rate (TDR) was defined as the ratio of tumor area in the mediastinal window to that in the lung window on HRCT. GGO lesions $\leq 20 \mathrm{~mm}$ in diameter on HRCT and those with a GGO ratio of $\geq 50 \%$ were included. These comprised pure GGO lesions, in which both the GGO ratio and TDR were $100 \%$, and mixed lesions. Chest images were acquired using a multidetector HRCT scanner that generated 64 1-mm slices. HRCT images were continuously acquired using the following parameters: section thickness: $1 \mathrm{~mm}$; pitch, 1 ; section spacing: $1 \mathrm{~mm}$; pixel resolution: $512 \times 512$; scanning time: $0.5 \mathrm{~s}$; and a high spatial frequency reconstruction algorithm with a $20-\mathrm{cm}$ field of view. Images were captured using mediastinal (level: 50 Hounsfield units [HU]; width: $350 \mathrm{HU}$ ) and lung (level: $-500 \mathrm{HU}$; width: $1800 \mathrm{HU}$ ) window settings.
Table 1 Pathological diagnoses of 114 lesions with a pure ground-glass opacity component

\begin{tabular}{lc}
\hline & Number $(\%)$ \\
\hline Preinvasive lesions & \\
Atypical adenomatous hyperplasia & $6(5)$ \\
Adenocarcinoma in situ & $70(61)$ \\
Minimally invasive adenocarcinoma & $16(14)$ \\
Invasive adenocarcinoma & \\
Acinar predominant & $2(2)$ \\
Papillary predominant & $10(9)$ \\
Invasive mucinous adenocarcinoma & $1(1)$ \\
Adenosquamous carcinoma & $1(1)$ \\
Benign tumor & $8(7)$ \\
\hline
\end{tabular}

All patients underwent video-assisted thoracic surgery (VATS) using 3 ports $(7,10$, and $12 \mathrm{~mm}$ ) without mini-thoracotomy. ${ }^{19,20)}$ Three-port VATS lobectomy with mediastinal lymph node dissection was indicated when lesions were $>15 \mathrm{~mm}$ in diameter or were diagnosed as invasive by intraoperative frozen section analysis. Threeport VATS wedge resection or segmentectomy was indicated when tumors were $\leq 15 \mathrm{~mm}$ in diameter and were diagnosed as noninvasive. HRCT-guided hook wire localization (Guiding Marker System; Hakko Medical Products, Tokyo, Japan) was used for 58 of 114 (51\%) pure GGO lesions and 25 of $77(32 \%)$ mixed GGO lesions. The Toranomon Hospital Institutional Review Board approved the protocol, and written informed consent was obtained from all patients.

Categorical variables were analyzed using the $\chi^{2}$ test. $p$-values less than 0.05 were considered statistically significant.

\section{Results}

Of 191 GGO lesions in 160 patients, 114 were pure and 77 were mixed. Histological diagnoses of the 114 pure GGO lesions included AIS $(n=70 ; 61 \%)$, MIA $(n=16$; $14 \%)$, invasive lung cancer $(n=14 ; 12 \%)$, atypical adenomatous hyperplasia (AAH; $n=6 ; 5 \%$ ), and benign tumors $(n=8 ; 7 \%$; Table 1), whereas those for the 77 mixed GGO lesions included AIS $(n=7 ; 9 \%)$, MIA or invasive adenocarcinoma $(n=58 ; 75 \%)$, lymphoma $(n=5 ; 6 \%)$, and benign tumors $(n=7 ; 9 \%)$. The number of mixed GGO lesions diagnosed as MIA or invasive lung cancer was greater than that of pure GGO lesions $(p<0.001$; odds ratio, 8.55). None of the 114 pure GGO lesions were associated with lymphatic and vascular invasion except one invasive adenocarcinoma (acinar predominant) lesion that was associated with lymphatic 
Table 2 Relationship between malignant tumors manifesting as pure GGO lesions and HRCT and PET findings

\begin{tabular}{|c|c|c|c|c|c|}
\hline & & \multicolumn{2}{|c|}{ Invasive lung cancer } & \multirow{2}{*}{ Odds ratio } & \multirow{2}{*}{$p$-value } \\
\hline & & + & - & & \\
\hline \multirow{2}{*}{ Pleural indentation } & + & 5 & 16 & 2.64 & 0.21 \\
\hline & - & 9 & 76 & & \\
\hline \multirow{2}{*}{ PET SUVmax $>0.8$} & + & 8 & 6 & 16.0 & $<0.001$ \\
\hline & - & 4 & 48 & & \\
\hline
\end{tabular}

GGO: ground-glass opacity; HRCT: high-resolution computed tomography; PET: positron emission tomography; SUVmax: maximum standardized uptake value
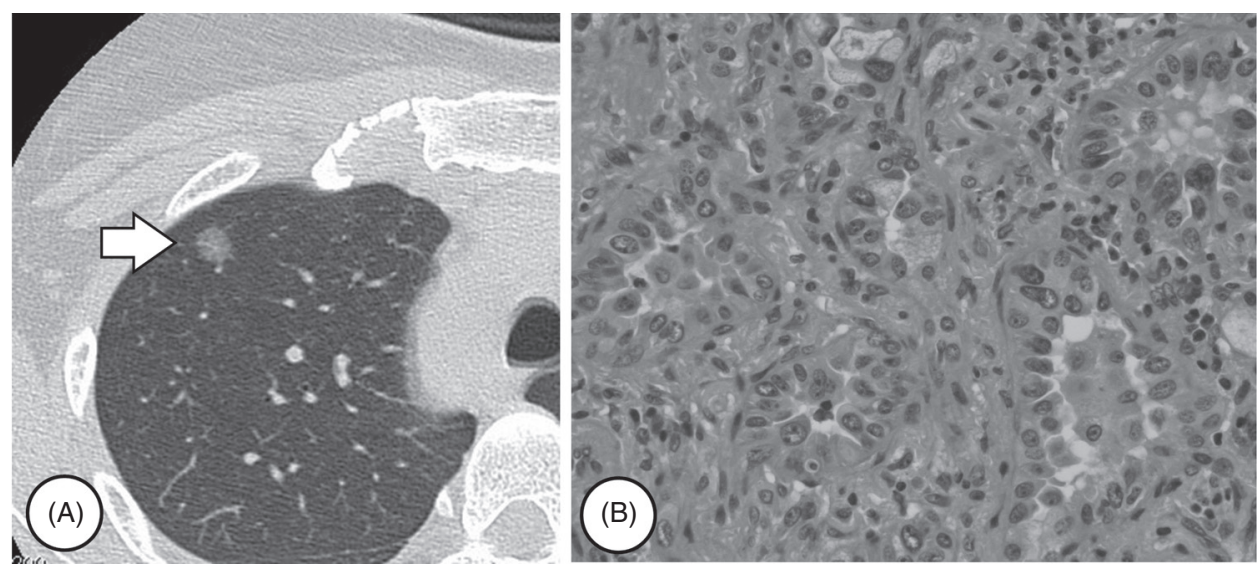

Fig. 1 (A) High-resolution computed tomography (HRCT) showing a 10-mm lesion with a pure ground-glass opacity (GGO) component and without pleural indentation (white arrow). (B) The pathological diagnosis is acinar-predominant invasive adenocarcinoma (haematoxylineosin staining; magnification, $\times 400)$.

invasion. Among the 77 mixed GGO lesions, 11 and two invasive adenocarcinoma lesions were associated with lymphatic invasion and vascular invasion, respectively. No lesion showed pleural invasion in both groups.

Of the 14 invasive lung cancers manifesting as pure GGO lesions, 13 were invasive adenocarcinoma (papillary predominant, $n=10$; acinar predominant, $n=2$; invasive mucinous, $n=1$ ) and 1 was adenosquamous carcinoma. All 16 patients with MIA exhibited stromal invasion in a papillary pattern (mean invasion depth, $2.9 \pm 1.0 \mathrm{~mm}$; range, $1.5-4.5 \mathrm{~mm}$ ).

After excluding the 8 benign lesions, 21 of the remaining 106 pure GGO lesions exhibited pleural indentation on HRCT. Of these, 5 were diagnosed as invasive adenocarcinoma, 4 as MIA, and 12 as AIS. Pure GGO lesions exhibiting slight pleural indentation on HRCT tended to be invasive lung cancer and not MIA, AIS, or AAH (odds ratio, 2.64; not significant; Table 2).

PET was performed for 66 pure GGO lesions. When the threshold SUVmax was set at 0.8 on the basis of receiver operating characteristic curve analysis, 8 of 14 lesions that exhibited PET positivity were diagnosed as invasive lung cancer. Pure GGO lesions that were positive on PET tended to be invasive lung cancer and not MIA, AIS, or AAH (odds ratio, 16.0; $p<0.001$; sensitivity, 67\%; specificity, 89\%; Table 2).

\section{Case Examples}

Case 1 was a patient with a $10-\mathrm{mm}$ pure GGO lesion in the right upper lobe of the lung, with no pleural indentation and an SUVmax of 0.9 on PET. The pathological diagnosis was acinar-predominant invasive adenocarcinoma, classified as Type E according to the Noguchi classification. ${ }^{1)}$ Segmentectomy with mediastinal lymph node dissection was performed (Fig. 1).

Case 2 was a patient with a 16-mm pure GGO lesion in the right lower lobe of the lung, with slight pleural indentation and an SUVmax of 0.9 on PET. The pathological diagnosis was MIA with papillary pattern 

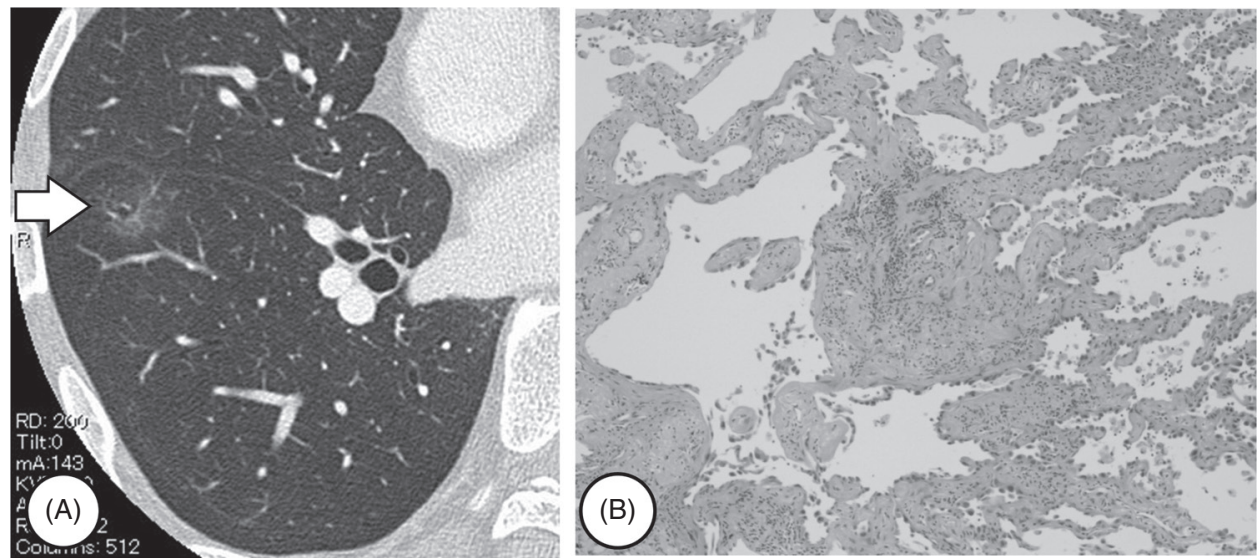

Fig. 2 (A) High-resolution computed tomography (HRCT) showing a 16-mm lesion with a pure ground-glass opacity (GGO) component and slight pleural indentation (white arrow). (B) The pathological diagnosis is minimally invasive adenocarcinoma with invasion in a papillary pattern (haematoxylin-eosin staining; magnification, $\times 400$ ).
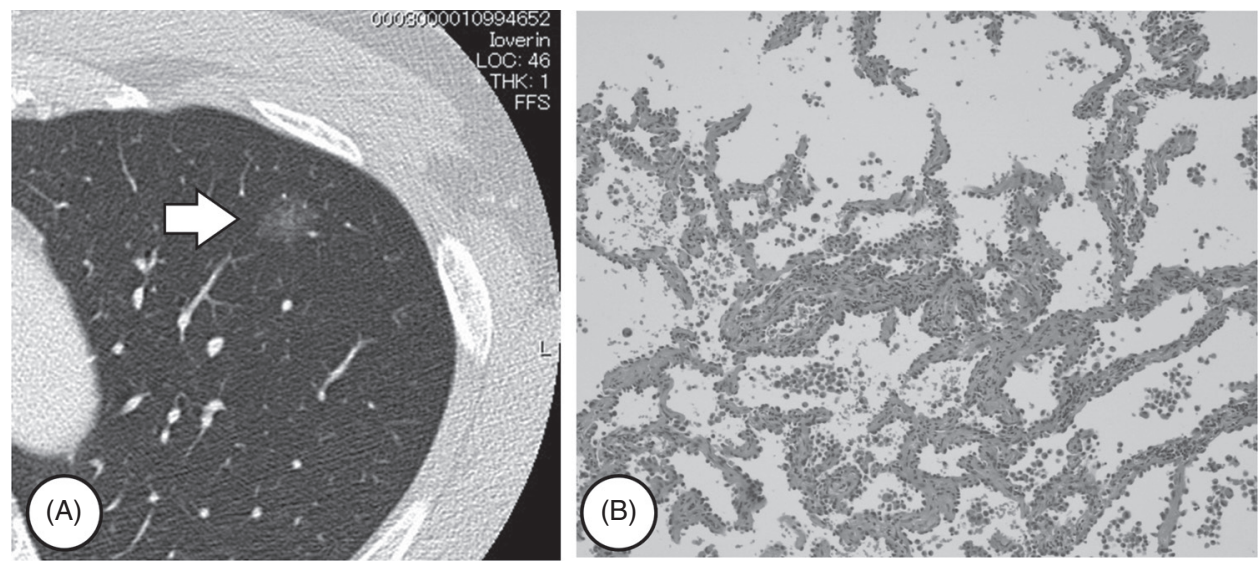

Fig. 3 (A) High-resolution computed tomography (HRCT) showing a 15-mm lesion with a pure ground-glass (GGO) opacity component and without pleural indentation (white arrow). (B) The pathological diagnosis is adenocarcinoma in situ (haematoxylin-eosin staining; magnification, $\times 400)$.

invasion, classified as Type $\mathrm{C}$ according to the Noguchi classification. Lobectomy with mediastinal lymph node dissection was performed (Fig. 2).

Case 3 was a patient with a $15-\mathrm{mm}$ pure GGO lesion without pleural indentation in the left upper lobe of the lung. PET was not performed for this patient. The pathological diagnosis was AIS, classified as Type A according to the Noguchi classification. Wedge resection was performed (Fig. 3).

\section{Discussion}

Of the total pure GGO lesions evaluated in this study, $12 \%$ were invasive lung cancers. However, we excluded tumors with $\leq 5 \mathrm{~mm}$ invasion designated as MIA; these accounted for $14 \%$ of the pure GGO lesions. Although the proportion of pure GGOs diagnosed as invasive lung cancers was smaller than that of mixed GGO lesions, it was not negligible. Suzuki, et al. ${ }^{9)}$ reported that $16 \%$ of pure GGO lesions, including MIA, were invasive adenocarcinomas.

Pure GGO lesions exhibiting pleural indentation on HRCT tended to be invasive lung cancer, albeit insignificantly. Pleural indentation does not occur when lesions are located far from the pleura. The problem of localization may lessen the reliability of pleural indentation as a risk factor for invasive lung cancer. However, pure GGO lesions positive on PET tended to be invasive lung 
cancer with a high specificity (89\%). It may be more reliable to propose surgical treatment when tumors exhibit even slight positivity on PET.

Although intraoperative frozen section analysis was performed for all cases, the pathologists and authors do not fully rely on the capacity of frozen section analysis to detect stromal invasion. When the frozen section analysis showed stromal invasion, lobectomy with mediastinal lymph node dissection was indicated. In many cases, however, the pathologists refrained from making clear comments about stromal invasion. We selected the type of resection mainly on the basis of imaging findings.

CT-guided hook wire localization was used for $51 \%$ pure GGO lesions. In our experience, of 500 procedures for 417 patients, systemic air embolism was the most serious complication, and it occurred in only 1 patient $(0.24 \%)$, albeit without any sequelae and with spontaneous resolution. ${ }^{21)}$

This study has several limitations. The results were influenced by selection bias in several phases, including patient referral to the Department of Thoracic Surgery, surgical treatment selection, and the decision to perform PET. Qualitative judgement of pure GGO lesions and pleural indentation is somewhat subjective. Quantitative analysis of tumor attenuation measured by 2- or 3-dimensional HRCT is required. Ikeda, et al. ${ }^{22}$ described HRCT histogram analysis using three-dimensional computerized quantification. Our impression was that lesions with thicker attenuation tended to be invasive lung cancers. Evaluation of PET results is also complicated. SUVmax varies considerably between instruments. Although we set the threshold at 0.8 on the basis of receiver operating characteristic curve analysis, it is not a universally acknowledged standard. Moreover, PET is not cost effective.

We are aware that the pathological diagnosis of invasive lung cancer never necessitates surgical treatment. To determine the indications for surgical treatment, a prospective cohort study with a long-term follow-up period is required. Prior to this, we propose that pure GGO lesions should be carefully monitored by periodic chest HRCT and that lesions with pleural indentation on HRCT or positivity on PET should be considered for surgical resection to ensure accurate pathological diagnosis and treatment. We have occasionally encountered patients with solid lung tumors in whom GGO lesions had previously been detected by HRCT, but were omitted from medical follow-up.

\section{Conclusion}

Of the pure GGOs examined, $12 \%$ were invasive lung cancers, most of which exhibited pleural indentation on HRCT or positivity on PET. Pure GGO lesions should be carefully monitored by periodic chest HRCT, and when they show pleural indentation on HRCT or positivity on PET, the option of surgical resection should be considered.

\section{Disclosure Statement}

The authors disclose no conflicts of interest.

\section{References}

1) Noguchi M, Morikawa A, Kawasaki M, et al. Small adenocarcinoma of the lung. Histologic characteristics and prognosis. Cancer 1995; 75: 2844-52.

2) Watanabe S, Watanabe T, Arai K, et al. Results of wedge resection for focal bronchioloalveolar carcinoma showing pure ground-glass attenuation on computed tomography. Ann Thorac Surg 2002; 73: 1071-5.

3) Sakurai H, Dobashi Y, Mizutani E, et al. Bronchioloalveolar carcinoma of the lung 3 centimeters or less in diameter: a prognostic assessment. Ann Thorac Surg 2004; 78: 1728-33.

4) Vazquez M, Carter D, Brambilla E, et al. Solitary and multiple resected adenocarcinomas after CT screening for lung cancer: histopathologic features and their prognostic implications. Lung Cancer 2009; 64: 148-54.

5) Yamada S, Kohno T. Video-assisted thoracic surgery for pure ground-glass opacities $2 \mathrm{~cm}$ or less in diameter. Ann Thorac Surg 2004; 77: 1911-5.

6) Yoshida J, Nagai K, Yokose T, et al. Limited resection trial for pulmonary ground-glass opacity nodules: fifty-case experience. J Thorac Cardiovasc Surg 2005; 129: 991-6.

7) Koike T, Togashi K, Shirato T, et al. Limited resection for noninvasive bronchioloalveolar carcinoma diagnosed by intraoperative pathologic examination. Ann Thorac Surg 2009; 88: 1106-11.

8) Travis WD, Brambilla E, Noguchi M, et al. International association for the study of lung cancer/ american thoracic society/european respiratory society international multidisciplinary classification of lung adenocarcinoma. J Thorac Oncol 2011; 6: 244-85.

9) Suzuki K, Asamura H, Kusumoto M, et al. "Early" peripheral lung cancer: prognostic significance of ground glass opacity on thin-section computed tomographic scan. Ann Thorac Surg 2002; 74: 1635-9.

10) Sakurai H, Maeshima A, Watanabe $S$, et al. Grade of stromal invasion in small adenocarcinoma of the lung: histopathological minimal invasion and prognosis. Am J Surg Pathol 2004; 28: 198-206. 
11) Borczuk AC, Qian F, Kazeros A, et al. Invasive size is an independent predictor of survival in pulmonary adenocarcinoma. Am J Surg Pathol 2009; 33: 462-9.

12) Yim J, Zhu LC, Chiriboga L, et al. Histologic features are important prognostic indicators in early stages lung adenocarcinomas. Mod Pathol 2007; 20: 233-41.

13) Maeshima AM, Tochigi N, Yoshida A, et al. Histological scoring for small lung adenocarcinomas $2 \mathrm{~cm}$ or less in diameter: a reliable prognostic indicator. J Thorac Oncol 2010; 5: 333-9.

14) Tsutani $Y$, Miyata $Y$, Nakayama H, et al. Prognostic significance of using solid versus whole tumor size on high-resolution computed tomography for predicting pathologic malignant grade of tumors in clinical stage IA lung adenocarcinoma: a multicenter study. J Thorac Cardiovasc Surg 2012; 143: 607-12.

15) Murakawa $T$, Konoeda $C$, Ito $T$, et al. The ground glass opacity component can be eliminated from the T-factor assessment of lung adenocarcinoma. Eur J Cardiothorac Surg 2013; 43: 925-32.

16) Okada M, Nakayama H, Okumura S, et al. Multicenter analysis of high-resolution computed tomography and positron emission tomography/computed tomography findings to choose therapeutic strategies for clinical stage IA lung adenocarcinoma. J Thorac Cardiovasc Surg 2011; 141: 1384-91.
17) Nakayama H, Okumura S, Daisaki H, et al. Value of integrated positron emission tomography revised using a phantom study to evaluate malignancy grade of lung adenocarcinoma: a multicenter study. Cancer 2010; 116: 3170-7.

18) Regier M, Derlin T, Schwarz D, et al. Diffusion weighted MRI and 18F-FDG PET/CT in non-small cell lung cancer (NSCLC): does the apparent diffusion coefficient (ADC) correlate with tracer uptake (SUV)? Eur J Radiol 2012; 81: 2913-8.

19) Ichinose J, Kohno T, Fujimori S, et al. Locoregional control of thoracoscopic lobectomy with selective lymphadenectomy for lung cancer. Ann Thorac Surg 2010; 90: 235-9.

20) Kohno T, Fujimori S, Kishi K, et al. Safe and effective minimally invasive approaches for small ground glass opacity. Ann Thorac Surg 2010; 89: S2114-7.

21) Ichinose J, Kohno T, Fujimori S, et al. Efficacy and complications of computed tomography-guided hook wire localization. Ann Thorac Surg 2013 Jul 26. [Epub ahead of print]

22) Ikeda K, Awai K, Mori T, et al. Differential diagnosis of ground-glass opacity nodules: CT number analysis by three-dimensional computerized quantification. Chest 2007; 132: 984-90. 